\title{
La enseñanza y su relación con el saber en los estudiantes universitarios colombianos'
}

\author{
Miguel Ángel Gómez Mendoza"
}

María Victoria Alzate Piedranhita"

\section{Resumen}

Se exponen los resultados de una investigación sobre las respuestas de los estudiantes a las exigencias de las formas de los saberes enseñados en la universidad desde la perspectiva de su relación con el saber. Estudio exploratorio, cualitativo, descriptivo y descriptivo en una muestra cualitativa con representatividad teórica de dieciséis estudiantes de seis cursos universitarios, que se desarrollaron en el segundo semestre de 2012 en la Universidad Tecnológica de Pereira, Colombia. Se adoptaron cinco dimensiones de la relación con el saber: (1) sentido; (2) dimensión de lo que es importante en el saber; (3) contrato didáctico; (4) relación de identidad y afectiva con el saber; y (5) actitudes de estudio. Tres rasgos generales surgen de la investigación: (1) si bien los estudiantes, aceptan en su relación con el saber la exigencia de la significación, esperan también los momentos de la designación; (2) obstáculo importante en la relación constituye la distancia entre lo que el curso propone y la representación que el estudiante puede tener de la práctica fuente y la práctica objetivo; (3) rasgo común para los cursos es la distancia entre la percepción de las exigencias del profesor antes y después de la evaluación. Se confirma la hipótesis comprensiva: la respuesta que dan los estudiantes a las exigencias de las formas de los saberes enseñados en la universidad dependen de su relación con el saber y de sus actitudes y tratativas o enfoques que esta relación implica.

\section{Palabras clave}

I- Artículo resultado del Convenio 708 de Saber - Relación con el saber - Educación superior - Enseñanza Estudiantes - Universidad.

2012 Ministerio de Educación Nacional de Colombia, Universidad Tecnológica de Pereira. Convocatoria Realización de estudios sobre Educación Superior 2012. (Código Vicerrectoría de Investigaciones, Extensión e Innovación de la Universidad Tecnológica de Pereira. VIIEUTP: 4-12-5. Código división financiera UTP: 5-11-3-234-25).

II- Universidad Tecnológica de Pereira, Pereira, Risaralda, Colombia.

Contactos: mgomez@utp.edu.co,

mvictoria@utp.edu.co 


\section{Teaching and its relation to knowledge among Colombian university students'}

Miguel Ángel Gómez Mendoza"

María Victoria Alzate Piedranhita"

I- This article is a result of Agreement 708 of 2012 Ministry of National Education of Colombia, Universidad Tecnológica de Pereira. Call Realización de estudios sobre Educación Superior 2012. (Code Vicerrectoría de Investigaciones, Extensión e Innovación de la Universidad Tecnológica de Pereira. VIIE-UTP: 4-12-5. Financial division code UTP: 5-11-3-234-25).

II- Universidad Tecnológica de Pereira, Pereira, Risaralda, Colombia.

Contact: mgomez@utp.edu.co, mvictoria@utp.edu.co

\section{Abstract}

This article presents the results of an investigation into student responses to the demands of the forms of knowledge taught at the university from the perspective of their relation to knowledge. It is an exploratory, qualitative, and descriptive study with a qualitative sample with theoretical representativeness of sixteen students from six university courses offered in the second half of 2012 at Universidad Tecnológica de Pereira, Colombia. Five dimensions of the relation to knowledge were adopted: (1) meaning, (2) dimension of what is important in knowledge, (3) didactic contract, (4) identity and affective relation to knowledge, and (5) study attitudes. Three general features have emerged from the research: (1) although students accepted the requirement of meaning in their relation to knowledge, they also expect moments of designation; (2) the distance between the course aims and the representation that the student may have of the source practice and target practice is a major obstacle in the relation; (3) the distance between the perception of the demands of the professor before and after evaluation is a common characteristic of the courses. The comprehensive hypothesis has been confirmed: the response given by students to the demands of the forms of knowledge taught at university depends on their relation to knowledge and the attitudes and negotiations or approaches that this relations implies.

\section{Keywords}

Knowledge - Relation to knowledge - Higher education - Education - Students - University. 
Algunos estudios han puesto en evidencia de manera global los procesos de diferenciación de las prácticas de estudio de los estudiantes universitarios, tanto en el plano de las estrategias de aprendizaje (ROMAINVILLE, 1993) como en el de las competencias lingüísticas y textuales (POLLET, 2001). El campo de de investigación que se exploró en esta ocasión es el de la relación que los estudiantes mantienen con las diferentes formas de saber que se les pide o demanda adquirir en la universidad. La noción de relación con el saber (rapport au savoir), ha tomado, desde hace veinte años, cada vez más importancia en el campo de las ciencias humanas y de la educación. Se trata de una noción que continúa en proceso de elaboración y que ha podido demostrar hasta ahora su real poder heurístico tanto por sus cuestionamientos, como por la relación con el campo de investigación e intervención que este concepto abre.

Desde los años 90 del siglo pasado, en la tradición de investigación sociológica, psicológica y de las ciencias de la educación francesas, un buen número de investigadores apelan a esta noción de relación con el saber. Ella permite una nueva aproximación al logro, al fracaso y a la deserción escolar y universitaria. Dos equipos de investigación de manera sistemática han hecho uso de esta noción en sus trabajos: el grupo de investigación del CREF (Centre de Recherche Education et Formation de la Universidad Paris X - Nanterre) y el grupo ESCOL (Education, Socialisation, et Collectivités Locales de la Universidad Paris VIII - Saint Denis).

Para Bernard Charlot (1997), promotor del segundo grupo

(...) la relación con el saber es el conjunto (organizado) de las relaciones que un sujeto humano (esto es singular y social) mantiene con todo lo que surge o se deriva del 'aprender' y del saber: objeto, 'contenido de pensamiento', actividad, relación interpersonal, lugar, persona, situación, ocasión, obligación, etc., asociados de alguna manera al aprender y al saber. (p. 22)
Según Jacky Beillerot (1998) integrante del grupo CREF, indagar la relación con el saber, es

(...) estudiar las situaciones donde se implementan los elementos de esta relación con el saber. Si se postula que la relación con el saber no es tanto un atributo como un proceso, él es entonces más accesible en situación, o a él el no es posible acceder sino en situación (provocada o natural). (p.7)

Para investigadores de este grupo como Beillerot; Blanchart-Laville; Mosconi (1996), la referencia a la teoría psicoanalítica está netamente marcada. La noción de relación con el saber es analizada a través de la problemática del deseo de saber. Es la dimensión clínica que fundamenta la coherencia epistemológica de sus investigaciones. Ahora bien, en una perspectiva teórica, la relación con el saber es percibida en un primer momento, en el nivel de su génesis y en términos de relación de objeto. Al respecto, como lo han desarrollado Beillerot; Bouillet; Blanchard-Laville; Mosconi (1989), Beillerot (1998) y Blanchard-Laville (1996), en sus trabajos de elaboración teórica y la relación con el saber, las concepciones de Wilfred Bion y Donald Wood Winnicott, que tratan de la construcción psíquica precoz de la capacidad de aprendizaje, del pensamiento y de las primera experiencias de saber se plantean en primer lugar, junto a la teoría de Jacques Lacan relacionada con el concepto de deseo, de deseo de saber y de su insatisfacción es, como tal, central en esta perspectiva.

Para Bernard Charlot, Élisabeth Bautier Jean-Yves Rochex (1992), investigadores del grupo ESCOL (Education, Socialisation, et Collectivités Locales de la Universidad Paris VIII - Saint Denis), la orientación de las indagaciones sobre la relación con el saber es más sociológica, incluso antropológica. Plantean la idea de una sociología del sujeto. Sus trabajos intentan dar una nueva dimensión a la cuestión del fracaso escolar en los niños 
y jóvenes provenientes de medios sociales desfavorecidos. Sus estudios se focalizan sobre la relación con el saber y sobre la relación con la escuela de los jóvenes que frecuentan las escuelas secundarias de los barrios periféricos de las ciudades francesas. Lo que se destaca en primer lugar, son las lógicas complejas $\mathrm{y}$ múltiples que subyacen en el trabajo escolar a través del análisis de aspectos como la relación con el lenguaje y relación con el saber y con el mundo de los estudiantes de secundaria (liceo) herederos de familias favorecidas y de nuevos estudiantes de liceo de familias populares. Para este grupo, la reflexión teórica se articula alrededor de la noción de sentido dado a la experiencia escolar y los procesos de identidad en la formación de personalidad. La perspectiva empírica se focaliza sobre los retratos biográficos de los jóvenes estudiantes de liceo de secundaria a partir de un balance de saber. Estos últimos se componen de producciones escritas sobre las expectativas de los alumnos de liceo frente a su escolaridad y sus saberes adquiridos, escolares o no escolares.

Es entonces en esta línea de estudios de este último grupo de investigadores (ESCOL, dirigido por Bernard Charlot), que se concibió esta investigación. Si bien, los estudios sobre la noción de relación con el saber implican, hasta ahora, solamente a la población de los alumnos de la escuela primaria y la educación secundaria francesa, en esta ocasión, se extiende el concepto de relación con el saber al ámbito de la educación superior o universitaria, para estudiar una población de estudiantes inscritos en la Universidad Tecnológica de Pereira-Colombia, con las precisiones y cuidados conceptuales y metodológicos pertinentes $\mathrm{y}$ propios del nivel superior o universitario del sistema educativo colombiano.

\section{Justificación del estudio}

En Colombia, según los datos del Ministerio de Educación Nacional de Colombia (2012):
En 2010, en todo el país hay 1.674.420 estudiantes en el sistema de la educación superior, de los cuales 1.587 .928 se encuentran matriculados en programas de pregrado (técnico profesional, tecnológico o universitario) y 86.492 en programas de posgrado (especialización, maestría o doctorado).

Y "La tasa de deserción por cohorte es de $45 \%$, es decir que de cada 100 estudiantes que ingresan a la educación superior, 55 eventualmente se gradúan, mientras 45 nunca lo hacen".

En consecuencia, según el Ministerio de Educación de Colombia (2009):

Uno de los principales problemas que enfrenta el sistema de educación superior colombiano concierne a los altos niveles de deserción académica en el pregrado. Pese a que los últimos años se han caracterizado por aumentos de cobertura e ingreso de estudiantes nuevos, el número de alumnos que logra culminar sus estudios superiores no es alto, dejando entrever que una gran parte de éstos abandona sus estudios, principalmente en los primeros semestres. Según estadísticas del Ministerio de Educación Nacional, de cada cien estudiantes que ingresan a una institución de educación superior cerca de la mitad no logra culminar su ciclo académico y obtener la graduación.

El complejo fenómeno social y educativo de la deserción y el fracaso y el logro académico en la educación superior colombiana en los dos primeros semestres o primer año universitario - que toca en Colombia prácticamente a uno de cada dos estudiantes - nos conduce a cuestionarnos: si bien en los últimos doce años, la educación superior colombiana conoce una masificación importante, ¿se puede hablar igualmente de una democratización de la enseñanza? El desafío es doble. Se trata, en 
primer lugar, de asegurar la cualificación, mediante aprendizajes de calidad, entre otros factores, esperada y sancionada por los diplomas universitarios, que se fundamente sobre la investigación o sobre la profesionalización. Pero el otro desafío, es también, asumir esta masificación preguntándonos sobre los mecanismos de fracaso que frenan la democratización asociadas a las maneras como los estudiantes se relacionan con los saberes que les ofrece la universidad.

En este contexto, surgen varias preguntas: ¿cómo el principal implicado, el estudiante aprendiz, vive esta situación y en particular sus aprendizajes? ¿Qué tipos de dificultades encuentra en esta relación con el saber universitario y cuáles son sus razones? Una abundante literatura (ROMAINVILLE, 1999, 2000; ALZATE; GÓMEZ, 2009, 2010a; ALZATE; DESLAULIERS; GÓMEZ, 2010), plantea diversas hipótesis que ponen en relieve los factores imputables al estudiante mismo. Estudios, como los de Alain Coulon (1997), exponen una correlación entre el origen sociocultural y las dificultades de los estudiantes para adaptarse a los códigos de la enseñanza universitaria: códigos lingüísticos, códigos asociados a los procesos de afiliación institucional y social, exigencias implícitas de la universidad, etc. Estas dificultades son entonces consideradas como el resultado de una falta o de un déficit: falta de motivación, falta de trabajo, falta de métodos, dominio insuficiente de la lengua materna, déficits cognitivos anteriores atribuidos a la enseñanza secundaria e incluso asociados a la categoría social.

Ahora bien, si estos estudios han podido explicar, en parte, el origen de un cierto número de dificultades encontradas por los estudiantes, parece que otras dimensiones respecto al fracaso y la deserción universitaria, examinadas en otras poblaciones estudiantiles universitarias, no han sido todavía suficientemente exploradas en la educación superior.

En consecuencia, para ayudar a los estudiantes universitarios en su proceso de logro académico y así contribuir a la superación de la deserción y abandono estudiantil, es necesario también estudiar la relación que ellos mantienen con el saber en general, de una parte; y con la naturaleza específica de los saberes enseñados en la educación superior o universitaria, de otra parte. De esta manera, en lugar de partir del postulado que las dificultades experimentadas o vividas por muchos estudiantes provienen de las diversas carencias, hemos sometido a prueba en este estudio el supuesto comprensivo según la cual estas dificultades son efectos de diferentes maneras de ser y especialmente de concebir el saber y acceder a él, esto es, de la relación con el saber.

\section{La importancia de estudiar el problema de la relación con el saber en los estudiantes universitarios}

El campo de investigación sobre la enseñanza de los saberes universitarios ha sido preocupación nuestra en los últimos cinco años (2009, 2010, 2010a, 2010b, 2010c) y parte, ante todo, de la hipótesis, según la cual para ayudar a los estudiantes universitarios es necesario también estudiar la relación que ellos mantienen con el saber en general, de una parte; y con la naturaleza específica de los saberes enseñados en la educación superior o universitaria, de otra parte. De esta manera, en lugar de partir del postulado generalmente aceptado, que afirma que las dificultades experimentadas o vividas por muchos estudiantes provienen de sus diversas carencias, se considera que el estudio de la relación con el saber ofrece una perspectiva diferente para su análisis (POZO; ECHERRÍA, 2009; HOUGARDY; PAMBU KITA, 1999).

En una investigación reciente, Enseñar en la Universidad. Saberes, prácticas y textualidad (ALZATE; GÓMEZ; ARBELÁEZ, 2011), nos limitamos de manera voluntaria a implementar las herramientas de análisis de las formas y sus contextos epistemológicos, textuales y didácticos de los saberes universitarios en 
algunas disciplinas académicas universitarias en las asignaturas o cursos de: didáctica del lenguaje, sociología de la educación, física, introducción a la filosofía, psicología del desarrollo. No obstante, desde entonces aparecía en el horizonte una pregunta que se abordó en esta investigación: ¿cómo la forma del saber influye sobre el logro del estudiante universitario? Interrogante que se responde desde la la teoría de la relación con el saber.

Una observación importante aparecía entonces en la investigación antes mencionada: en la operación compleja que es la práctica de enseñanza en la educación superior, el profesor intenta hacer acceder a los estudiantes ya sea a una práctica de investigación o a una práctica profesional. Se constató que esta transmisión universitaria plantea un cierto número de problemas. No existe una transmisión de una práctica en el sentido de una receta que haría a los estudiantes competentes, sino más bien enfoques o tratativas de enseñanza que exigen de estos últimos tomar en cuenta una serie de obligaciones con mucha frecuencia implícitamente declaradas: comprender los conceptos que no están claramente explícitos en el curso, enfrentamiento de saberes no estables y problemas específicos de una profesión, etc.

La siguiente pregunta que surgió entonces en este marco, y que ahora orienta este artículo de investigación, se plantea así: ¿responder a las exigencias de las diferentes obligaciones de la enseñanza universitarias implica una cierta forma de relación con el saber, él mismo asociado a una relación con el mundo (una manera de vivir, ciertas actitudes ante el saber y el estudio universitario)?

Este interrogante nos condujo entonces a una serie de preguntas complementarias que se respondieron en la investigación: (1) ¿Qué relaciones mantienen, de manera general, los estudiantes, con el saber universitario? Esta pregunta se plantea a partir del postulado, según el cual, esta relación con el saber está determinada por una cierta manera de vivir, por ciertas actitudes y por un cierto tipo de relación con el mundo. (2) ¿Cómo los estudiantes perciben ciertas características de los saberes enseñados: formas de situación didáctica, elementos asociados al grado de problematización y de sentido de los enunciados, tipos e importancia de razonamientos específicos a ciertos cursos universitarios, relación afectiva con el saber, dificultades asociadas a las actitudes frente al estudio universitario y de contrato didáctico? (3) ¿Cómo interactúan los estudiantes con las características antes indicadas del saber universitario? (4) ¿Cómo perciben los estudiantes las actividades que se les pide cumplir y que ponen en juego o implementan para llevarlas a cabo?

En este contexto, el enfoque descriptivo y comprensivo de la investigación realizada en ese entonces sobre la naturaleza de los saberes universitarios y sus procesos de transmisión, se examina, en esta ocasión, a través de la descripción de la forma que asume la relación con el saber de los estudiantes en la Universidad Tecnológica de Pereira, Colombia.

\section{Las cinco dimensiones de la relación con el saber: una} aproximación inicial

Se reitera, que relación con el saber se analizó a través de cinco dimensiones susceptibles de generar problemas o dificultades de aprendizaje ${ }^{1}$ universitario en los estudiantes universitarios para estudiar, a saber: (1) la dimensión del sentido; (2) la dimensión de lo que es importante en el saber; (3) el contrato didáctico; (4) la dimensión de la relación de identidad y afectiva con el saber; y (5) las actitudes de estudio. Se trató entonces de explorar en una muestra cualitativa con representación teórica de estudiantes universitarios, la manera cómo estos se relacionan con el saber que se ofrece en un cierto número número de cursos universitarios a través de las cinco dimensiones

1- Aquí la expresión dificultades de aprendizaje no debe entenderse en el sentido clínico y psicológico de grupo heterogéneo de alteraciones que se manifiestan en dificultades en la adquisición y uso de habilidades de escucha, habla, lectura, escritura, razonamiento o habilidades matemáticas. 
enunciadas, y que buscan dar cuenta de la relación con el saber de los estudiantes, así como de algunas dificultades y situaciones de fracaso que podrán resultar o surgir en el desarrollo de los cursos universitarios.

Veamos a continuación una breve definición de estas dimensiones:

(1) El sentido de los conceptos y de los enunciados. Se aborda esta cuestión, retomando de Gilles Deleuze (1990), la distinción que estableció entre la significación y la designación en la producción de sentido de un discurso universitario. En la educación universitaria, el discurso de la enseñanza se presenta con frecuencia como un texto. Con frecuencia, es un texto constituido de diferentes elementos: el discurso oral del profesor durante los cursos o sesiones, los soportes o apoyos proyectados en pantalla; el programa del curso, los documentos o las lecturas a las cuales los estudiantes son remitidos. Se trata, entonces, como afirma Bernard Rey (2002, 2005), de un texto, porque el conjunto de lo que es formulado tiene una coherencia (incluso si el texto es constituido de elementos dispersos). Igualmente, las palabras que constituyen estos enunciados no designan en general, las cosas exteriores que se podrían ver o tocar, ellas tienen sus sentidos y sus relaciones mutuas en el marco del texto.

Entonces, el sentido de los discursos no se deriva ya de la relación que mantienen los enunciados con una realidad inmediata y concreta compartida entre los locutores universitarios - profesor y estudiantes - sino que emerge de la relación entre estos enunciados. En oposición, en la vida corriente, los enunciados sacan su sentido del hecho que ellos refieren a los objetos o a las acciones que constituyen el ambiente actual y familiar de los locutores. La palabra está entonces anclada en la situación del momento. Para expresar esto, se dice que el sentido del discurso del profesor, tal como él se presenta en la educación superior, nace no de la designación, sino de la significación.

(2) Lo que es importante en el saber. Se adopta la hipótesis de Èlisabeth Bautier \&
Jean-Yves Rochex (1998), quienes consideran que un gran número de dificultades en los estudios residen en la relación de "evidencia y de adherencia al lenguaje y a la experiencia que se tiene con él" (p. 6). Esta relación implicaría resistencias de reconocimiento del carácter construido del saber, e incluso, de reconocimiento de las formas de lenguaje simbólicas y discursivas que lo constituyen, en este caso, en la enseñanza universitaria.

(3) El contrato didáctico. Guy Brousseau (1980) y Franc Morandi \&t René La Borderie (2006), consideran la noción de contrato y anotan que el carácter explícito de la implementación de un situación compromete a diferentes personas. Un contrato es firmado deliberadamente por las diferentes partes. En el contrato didáctico, se teje así una relación que determina lo que cada asociado, en este caso, el profesor y el estudiante universitarios, tendrá la responsabilidad de administrar y de la que será de una u otra manera, responsable frente al otro.

(4) La relación de identidad y afectiva con el saber. El lenguaje en la enseñanza universitaria puede presentarse bajo una pluralidad de registros de enunciación. Las formas textuales empleadas pueden mostrar un estado de diferentes posturas que tiene el sujeto con el saber: el yo-mí de la experiencia vivida, el del relato, el que analiza y argumenta, etc. Aquí, se plantea como hipótesis que los estudiantes universitarios pueden tener problemas para pasar fácilmente de un registro al otro, o peor aún, pueden tener la tendencia a privilegiar el registro lingüístico yo-mi de la experiencia familiar y subjetiva.

(5) Las dificultades asociadas a las actitudes de estudio. Con el vocablo estudio, se reúnen diversas actividades: la asistencia y las actividades del estudiante en los cursos universitarios, incluyendo aquellas que lleva a cabo fuera del local del curso y, en particular, la búsqueda de informaciones complementarias y sus actividades concretas para aprender. Cuando se pregunta a los estudiantes sobre sus prácticas de estudio y las maneras como ellos se movilizan, sus respuestas remiten a la hipótesis, 
según la cual, ellas están asociadas a una cierta concepción del saber. Este concepción puede entonces tener un impacto sobre el éxito y logro universitario del estudiante.

\section{Metodología²}

La metodología empleada se identifica con un enfoque descriptivo y comprensivo de los cursos universitarios, en esta ocasión complementado, con una descripción de la forma de la relación con el saber de los estudiantes.

$\mathrm{Al}$ respecto Mattew B. Miles y Michele Huberman (2003), consideran que

Los investigadores cualitativos trabajan con pequeñas muestras de personas, que viven en una situación familiar (en su 'nicho') en su contexto y se estudian en profundidad - a diferencia de los investigadores cuantitativos que buscan múltiples casos descontextualizados y apuntan a una representatividad estadística. (...) El tipo de muestra llamado 'representatividad teórica' busca encontrar los ejemplos de un constructo teórico y de esta manera elaborarlo y examinarlo. (p. 59-60)

Ahora bien, según De Ketel \&t Roegiers (2009), se puede afirmar que

(...) una entrevista puede ser libre, semidirigida o dirigida: - ella será llamada libre cuando el entrevistador se abstiene de plantear preguntas que apuntan a reorientar la entrevista; - ella será llamada dirigida cuando el discurso de la persona entrevistada constituye exclusivamente la respuesta a las preguntas preparadas

2- El tipo de entrevistas en esta investigación tiene esencialmente un enfoque exploratorio y cualitativo y se constituyó en punto de partida para otra investigación en marcha para construir, con más agudeza, un cuestionario dirigido al conjunto de la población de estudiantes implicados en los cursos que serán objeto del proyecto que se propone inicialmente. Se podrá entonces, en un segundo momento de la investigación (enfoque confirmativo y cuantitativo), validar la construcción de algunas dimensiones de la relación con el saber de los estudiantes y el repertorio de dificultades encontradas en una población más amplia. de antemano y planificadas en un orden preciso; - ella será llamada semi-dirigida cuando el entrevistador prevé algunas preguntas a plantear como puntos de orientación. (p. 13-14)

\section{En consecuencia, según los autores}

(...) una entrevista semi-dirigida se identifica por la presencia de dos características: - produce de parte del entrevistado un discurso que no es lineal, lo que significa que el entrevistador orienta la entrevista en ciertos momentos; - las intervenciones del entrevistador no están siempre previstas de antemano. A lo más, este prevé algunas preguntas importantes, o algunos puntos de orientación (p.146)

En consecuencia, y manteniendo una continuidad en el tema y campo de investigación antes indicado, limitamos este nuevo examen de la enseñanza universitaria en términos de relación con el saber, a una muestra cualitativa con representatividad teórica de estudiantes que asistieron durante el segundo semestre de 2012 a los siguientes cursos universitarios, como lo indica el cuadro.

Cuadro 1 - Muestra cualitativa con representatividad teórica de estudiantes entrevistados según cursos universitarios.

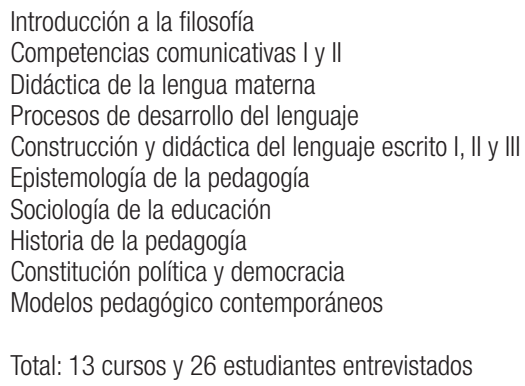

Fuente: Oficina de Registro y Control de la Universidad Tecnológica de Pereira, Colombia, 2012

Teniendo en cuenta el objetivo del estudio, explorar la relación con el saber de los 
estudiantes de los cursos indicados en el Cuadro 1 , se concibieron y aplicaron los siguientes procedimientos: (1) Realización de entrevistas semi-directivas o semi-dirigidas con cada uno de los estudiantes universitarios de la muestra. La entrevista semi-dirigida tuvo los siguientes objetivos: (a) discernir, en una perspectiva global, la relación con el saber del estudiante, esto es, en una perspectiva biográfica; (b) abordar las dificultades que tienen los estudiantes asociadas a las cursos que siguen y a su manera de estudiar; (c) desde una perspectiva más sistemática, indagar por las maneras como los estudiantes conciben y practican el estudio con miras a la presentación de exámenes parciales y finales, y sus reacciones a este tipo de evaluaciones. (2) Obtención de información complementaria y matizada mediante un escrito que se les solicitó a los estudiantes: un balance de saber, como lo sugiere Élisabeth Bauthier y Jean-Yves Rochex (1998) con los estudiantes de la educación secundaria francesa, obviamente, con sus ajustes correspondientes a la especificidad de la enseñanza superior o educación universitaria. De esta manera, se propuso a los estudiantes redactar un corto texto con el objetivo de responder a dos preguntas o cuestiones: (a) ¿Aprender, es....?; (b) ¿Desde que usted nació, que ha aprendido, que es lo importante para usted? En principio ninguna otra recomendación y precisión se plantea para este balance de saber, con el fin de no influir ni sobre la manera de abordar o enfocar las respuestas a los asuntos asociados, ni sobre la manera de redactar de los estudiantes universitarios a los cuales se les solicitará su colaboración para obtener esta información sobre su relación con el saber.

\section{Resultados}

Visto en perspectiva, para el estudiante surge una especie de determinación preexistente al entrar y empezar en los estudios universitarios. La explicación sociológica enfatiza el factor de pertenencia o proveniencia socio-cultural, los estudiantes que vienen de medios desfavorecidos consideran que tienen más riesgo y están expuestos al fracaso. Otra corriente de estudios, explica las dificultades en el logro de un estado de déficit en los métodos de trabajo, lagunas en el dominio de la lengua materna y los lenguajes especializados que se usan en la universidad.

En la investigación realizada, se exploró una vía de explicación poco estudiada en la enseñanza universitaria: algunas de las dificultades que debe superar el estudiante universitario para tener éxito tienen que ver con lo que acontece y hace en los cursos a los que asiste. Las dificultades, probablemente tan diversas, visibles o ocultas, obstaculizarían la transmisión del saber del profesor al estudiante, y serían provocadas especialmente por la naturaleza específica de los saberes universitarios enseñados. La singularidad de la relación con el saber del estudiante se convirtió entonces en objeto de indagación.

Con un intencionado objetivo de generalización, se agrupan u organizan las constataciones y resultados. Según algunos de los elementos que constituyen o identifican las cinco dimensiones de la relación con el saber adoptadas en el estudio.

\section{La relación con el saber específico}

¿Qué es importante en el saber? Las respuestas de los estudiantes son testimonio de un interés por la validación del saber universitario enseñado, aceptando su carácter construido e inestable. También declaran privilegiar los razonamientos a los resultados, aceptar la revisión de nociones y conceptos vistas en los cursos cuando aparecen nuevas teorías (presencia de la práctica fuente). La importancia de tomar en cuenta de la práctica objetivo de manera concreta en los cursos universitarios se siente o reclama en las respuestas obtenidas. De este modo, algunas respuestas a las preguntas abiertas son evidencia de la búsqueda de la posible utilidad de los cursos en un plano o 
marco profesional. Los estudiantes coinciden en la importancia de tomar en cuenta su futuro profesional en los cursos.

Si bien los cursos que siguieron los estudiantes se distinguen por su objeto de estudio, ciertos aspectos considerados como posibles fuentes de dificulta no cambian tanto. Un sector de estudiantes juzga esencial las demostraciones conceptuales en la comprensión general de los cursos. Sin embargo, estas exposiciones y demostraciones conceptuales aparecen a menudo como complicadas de seguir. Enfrentar esta dificultad implica recurrir a los ejemplos, las ilustraciones con el riesgo de alejar a los estudiantes del acceso a la teoría científica del saber sabio.

\section{Las prácticas fuente y objetivo}

En la educación superior, los profesores intentan iniciar a sus estudiantes en la práctica que se encuentra en el origen del saber que ofrecen, es decir la práctica de investigación. Es a esta que se denomina-la práctica fuente. Los profesores de universidad lo hacen porque es justamente su especificidad de ser a la vez investigadores $\mathrm{y}$ profesores, productores $\mathrm{y}$ difusores de saberes. Tienen la convicción que un curso no consiste solamente en presentar los resultados de la ciencia, sino también en hacer conocer las operaciones que han llevado a esos resultados. Un saber significativo, no se limita al conocimiento de una continuidad de enunciados considerados como verdaderos, exige que se lleven a cabo los procedimientos que permiten afirmar que ellos son verdaderos. Idealmente, el profesor debería compartir con los estudiantes una práctica de investigación. Pero para familiarizar a los estudiantes en esta práctica, el profesor solo dispone de su discurso, es decir de un texto, lo que no siempre es suficiente. Se derivaría de aquí entonces una situación propia de la enseñanza universitaria: (a) el profesor quiere que los estudiantes no se contenten con escuchar (o leer) el texto del saber, sino que también se apropien de los problemas científicos que allí están presentes, que se planteen y reconstituyan por sí mismos las actividades intelectuales que han conducido al saber. (b) Los mismos estudiantes, tienen la tendencia a apoyarse en el contrato didáctico, el cual puede enunciarse así: "Lo que el profesor exigirá en el examen debe habérsenos enseñado durante su curso". Forzando el asunto, se podría decir que la exigencia de los estudiantes al profesor es la siguiente: "Díganos directamente lo que usted quiere que digamos o que hagamos en el examen". (c) Cuando el profesor rechaza acceder a esta exigencia. Quisiera que los estudiantes realizaran el trabajo intelectual que conduce al saber y que hasta cierto punto lo reconstruyeran. La tarea de establecer el saber es devuelta por el profesor a los estudiantes. Es lo que se llama la devolución.

La devolución, entonces es una actividad que en el proceso pedagógico y didáctico, en este caso universitario, va en una dirección casi inversa para que el saber sea pensado personalmente por el estudiante; la devolución debe integrarse a la actividad de pensamiento personal o individual.

Ahora bien, en la enseñanza universitaria, el texto del saber lleva la marca de otra práctica diferente a la práctica fuente. Se trata de la práctica profesional en la que desemboca muchos de los curso seguidos por el estudiante. Es lo que se llama la prácticaobjetivo. Ella aparece, en el caso de la enseñanza superior, con una clara orientación de profesionalización. También está presente en las clases universitarias, para las cuales una salida profesional es siempre el horizonte preciso (derecho, medicina, administración, psicología, ciencias aplicadas, etc.). Ahora bien, como se ha visto, es difícil familiarizar al estudiante en una práctica (la profesión) a través de un texto (el saber). Porque un saber cubre un campo de fenómenos estrechamente circunscrito mientras que una práctica profesional se ejerce en un medio en el cual los fenómenos de naturaleza múltiple se producen. De este modo, un curso de física ofrecido a un futuro ingeniero, trata de 
los fenómenos que han sido delimitados por la construcción textual que se llama ciencia física. Pero el ingeniero deberá atender, en el ejercicio de su profesión, las determinaciones que serán físicas, pero también industriales, económicas, organizacionales, humanas, sociológicas, etc., y esto de manera simultánea.

Los estudiantes están interesados tanto en la práctica fuente como en la práctica objetivo en la construcción y desarrollo de los cursos. Esta constatación se confirma además en las respuestas obtenidas. En efecto, si un número importante de estudiantes evocan positivamente las discusiones y debates, que tienen lugar durante el curso, otros insisten sobre el interés de los conocimientos adquiridos durante los cursos para su futura profesión. No obstante, los estudiantes consideran que la referencia explícita a la práctica fuente en un curso podría derivar en otro tipo de estudio, muy extenso para ser desarrollado en este escenario. Cuando la práctica objetivo está presente según los estudiantes entonces podría impedir comprender el interés de las materias universitarias por sí mismas, y acabar por considerarlas únicamente como herramientas de utilidad inmediata.

\section{La relación de identidad y afectiva con el saber}

Se observa que en la medida en que se desarrollan los semestres universitarios, una mayoría de estudiantes reconoce haber cambiado su mirada sobre el saber en general, así como la disciplina involucrada de manera más específica. Parece entonces que los estudiantes se dan cuenta de las transformaciones que se producen en su identidad intelectual y en el plano de las relaciones con sus pares e integrantes de sus familias, es decir, admiten la transformación de su yo intelectual y las relaciones que establecen en su vida universitaria. Igualmente, los estudiantes se distancian de sus convicciones que tienen un tinte egocéntrico y comienzan a ver nuevas perspectivas y puntos de vista. Se agrega en este contexto, que un sector de estudiantes consideran al profesor y su enseñanza como factores determinantes en sus prácticas universitarias de saber.

\section{Las actitudes de estudio}

Algunos estudiantes afirman trabajar sus cursos cerca del fin de los semestres, otros insisten sobre la importancia de estudiar desde el comienzo, familiarizarse tempranamente con el curso y seguirlo de manera sistemática. Los análisis realizados permiten destacar o desvelar una situación un tanto paradójica. En efecto, si la mayoría de los estudiantes considera importante intentar encontrar las relaciones entre las ideas, conceptos y nociones que pertenecen a dominios o campos diferentes, otros prefieren estudiar cada parte del curso paso a paso. Las entrevistas indican que los estudiantes se dedican a comprender las nociones a medida que avanza el curso. Sin embargo, esta etapa podría llegar a veces más tarde que los exámenes parciales o finales, $\mathrm{y}$ entonces es necesario que los estudiantes entren en una segunda fase de estudio: la búsqueda de las relaciones y vínculos entre ideas, conceptos y nociones ofrecidos en la enseñanza universitaria. Un sector de estudiantes manifiesta comenzar el estudio para el examen acudiendo a los resúmenes y apuntes de curso sintéticos, manera parcial de estudiar que podría interpelar a los cursos universitarios que se exponen por un encadenamiento rigurosamente lógico porque los estudiantes perderían los eslabones de la cadena de razonamiento general del curso.

\section{El sentido de los conceptos y enunciados}

Si bien los estudiantes no señalan necesariamente la necesidad y posibilidad de dominar todos los enunciados del profesor de manera inmediata, sin embargo, muchos de ellos declaran la exigencia de la designación. Dicho de otra manera, para comprender una noción, concepto, teoría, tienen necesidad 
de concretizarla en ejemplos. Esta demanda se presenta cuando no hay una comprensión inmediata del sentido de una noción y los estudiantes desean ver el saber contextualizado por un enfoque más concreto y explicado en un lenguaje familiar.

El creciente interés por los cursos universitarios es un factor que contribuye a la aclaración de los nociones que en los inicios aparecen difíciles o incomprensibles. Sin embargo, cuando los cursos tienen una orientación didáctica más explícita los estudiantes no sugieren o exigen este proceso de traducción a un lenguaje más familiar a ellos. Se reitera que en ocasiones para diversas disciplinas universitarias los estudiantes privilegian la designación y se aferran a lo concreto para comprender, pero lo concreto puede implicar diferentes niveles. Este puede destacar los fenómenos de la vida corriente o las experiencias de las ciencias, como también las nociones de los cursos convertidas en objetos de referencia.

Algunos estudiantes privilegian la significación y las relaciones entre las diferentes proposiciones de las disciplinas objeto de estudio de los cursos. Estos son los mismos que manifiestan la importancia y necesidad de que los profesores acudan simultáneamente a la designación y a la significación para darle un sentido más profundo a sus aprendizajes. No obstante, parece que establecer las relaciones entre las diversas proposiciones de un curso puede tener dos orígenes: de una parte, los estudiantes comprenden estas relaciones porque se les fueron explicadas acercándolas a la designación, y de otra parte, son ellos mismos quienes las determinan analizando desde su propia iniciativa antes de leer los documentos recomendados en el curso. Serían estos los estudiantes autónomos que darían un sentido verdadero a sus cursos.

Se puede hablar de una figura de suspensión del sentido en los cursos universitarios. Los estudiantes esperan que los desarrollos posteriores que se dan en el curso contribuirán a una mejor comprensión, situación que puede generar una actividad intelectual favorable a la construcción de sentido. Sin embargo, esta construcción o elaboración se apoya sobre el establecimiento de relaciones entre diferentes enunciados. Cuando algunas justificaciones se omiten para simplificar el curso, relaciones lógicas más profundas $\mathrm{y}$ complejas se pueden perder. Esta suspensión del sentido no podría conducir a una construcción posterior. En consecuencia, la presentación de ejemplos tiene sus riesgos. De un lado, permite comprender a través de un enfoque más concreto, más inmediato; y de otro lado, podría impedir la elaboración de las relaciones de sentido que existen en las teorías científicas. Intentar reducir un obstáculo que se encuentra en los estudiantes podría a veces generar otro.

\section{El contrato didáctico}

La idea de contrato, sugiere el carácter explícito de la creación de una situación que involucra varias personas. Un contrato está firmado deliberadamente por diferentes partes. En el contrato didáctico, se construye de esta forma una relación que determina lo que cada una de las partes, el profesor y el estudiante, tendrá la responsabilidad de gestionar o administrar y como cada uno será responsable frente al otro. Se construirá entonces entre enseñante y enseñado una serie de expectativas más o menos recíprocas que entrañaran $\mathrm{y}$ legitimaran ciertos comportamientos.

Los estudiantes perciben la manera como el curso es orientado cuando el profesor manifiesta qué espera de ellos en los exámenes; es decir, cuando no hay un malentendido respecto a la evaluación. De este modo, si el curso universitario se presenta claramente estructurado, y si la forma de evaluación de formula de manera explícita, los estudiantes tampoco están exentos de las dificultades propias del estudio de las materias universitarias. De hecho, la comprensión de ciertos conceptos y la memorización de una terminología percibida como abundante $\mathrm{y}$ 
extensa solo aparecerá cuando se entra en el estudio de las relaciones entre nociones. De manera inversa, la evaluación puede sorprender a los estudiantes que privilegian una estrategia de estudio en función de una problematización fuerte del curso, mientras que los exámenes parciales y finales se elaboran por el profesor con un modelo de restitución de conocimientos.

Los estudiantes afırman distinguir con relativa facilidad, en el discurso del profesor, lo que es esencial y lo que es anecdótico, un número significativo de ellos saben muy bien lo que se espera de ellos en los exámenes. El cuestionamiento de los estudiantes sobre los exámenes o la evaluación podría tener dos fuentes: una, la distancia entre lo que fue anunciado por el profesor (con frases incompletas) y los exámenes con preguntas abiertas para orientar su estudio que se mencionarían en el programa o en ciertos momentos del curso y que a la hora de la evaluación inducirían al error a los estudiantes; otra, sería el contraste entre el contexto agradable del curso y la exigencia asociada necesariamente a la prueba de evaluación o examen. Si los estudiantes piensan que en algunos cursos cada uno puede tener su opinión y percibe el examen como una formalidad como consecuencia de la vivencia distendida de las sesiones del curso, el riesgo del fracaso parece evidente.

También, la evaluación de los cursos universitarios puede interesar a los estudiantes cuando los exámenes se elaboran teniendo como horizonte su futura profesión, en este caso, las preguntas serían ante todo de orden técnico-instrumental. Sin embargo, es necesario en este tipo de evaluación que el profesor comunique oportunamente estas exigencias para que se establezca de antemano el contrato didáctico necesario.

\section{Problematización}

Si la devolución no se logrará verdaderamente sino en las situaciones exteriores a las sesiones de cursos universitarios propiamente dichas, no se excluye, obviamente, que pueda haber, en el marco de un curso de forma tradicional (curso magistral), episodios de devolución. Uno de los medios para que esto suceda es que el profesor exponga el saber bajo una forma problematizada. Esto sucede de manera práctica cuando en el marco de su discurso, los problemas se planteen para que los estudiantes puedan apropiarlos, es decir, para que puedan intentar responderlos. Dicho de otra manera, se trata que el saber no se presente solamente bajo la forma de enumeración de resultados, sino que los resultados aparezcan como respuestas a los problemas previamente planteados. Se podría decir, que en oposición a la problematización, se encontraría, por ejemplo, la enumeración (sin que esta última sea la única forma opuesta a la problematización). Entre más un curso se aproxime a la forma de enumeración, como sucesión de elementos presentados en una serie, como única razón de su articulación común, entonces menos la dimensión problemática estará presente. No habría en la enumeración, el hilo conductor que le da sentido, y que permite de esta manera a los estudiantes, volver a apropiarse del saber a través de un pensamiento propio. La presentación del saber como respuesta a los problemas, que constituye otra manera de devolución, presenta también la ventaja de ser análoga o semejante a lo que sucede en la práctica científica. El investigador es alguien que pasa su tiempo intentando resolver los problemas. De esta forma, una presentación problematizada del saber parece a primera vista, garantizar que habría devolución, y que los estudiantes darían sentido al saber. En realidad, las cosas son más complejas y puede haber formas de problematización del saber que no generen la devolución, porque los problemas son formulados de tal forma que el estudiante no pueda apropiárselos o que le aparezcan como arbitrarios. En consecuencia, no se puede entonces evitar la pregunta por las condiciones para que los problemas del saber tengan sentido para los estudiantes. 
Cuando en los cursos los estudiantes se comprometen con la significación dan más importancia o prioridad a los razonamientos y a los debates contradictorios. De esta manera, se constata que entre los estudiantes que declaran encontrar con el paso del tiempo un creciente interés por sus cursos se hallan también aquellos que dan importancia a la problematización de las materias universitarias.

Ahora bien, en la universidad los estudiantes siguen con interés los cursos donde se realizan diversos ejercicios o trabajos prácticos. Estos ofrecen una preparación para los exámenes y una aproximación las materias o disciplinas universitarias más concretas que la de los cursos teóricos. Sin embargo, esta aproximación debe ser matizada, porque existe una diferencia esencial, que los estudiantes podrían no percibir, entre la resolución de un problema que hace competentes a los estudiantes en una materia aprendida y la situación-problema que consiste en volver el saber problemático; también aquí los estudiantes no podrían percibir que la teoría abordada en el curso es necesaria para continuar el desarrollo del curso y el de los ejercicios.

\section{Conclusiones y consideraciones generales}

Más allá de las dificultades que parecen desprenderse de la naturaleza propia de las disciplinas universitarias, tres rasgos comunes y generales surgen de la investigación.

El primero, no se identifica con una de las hipótesis iniciales que consideraba que el estudiante inscribe totalmente su actividad ya sea en una lógica de significación o en una lógica de designación. Cualquiera que sea el campo de estudio universitario, parece que si bien los estudiantes, parecen aceptar la exigencia de la significación de los estudios superiores, esperan sin embargo los momentos de la designación. Las explicaciones, las ilustraciones, los ejemplos le son necesarios.

El segundo rasgo relevante es el obstáculo importante que constituye la distancia entre lo que el curso propone y la representación que el estudiante puede tener de la práctica fuente y la práctica objetivo. Para los estudiantes los cursos con orientación teórica se distancian de la práctica objetivo y los cursos con orientación profesional de la práctica fuente.

El tercer rasgo común para los cursos con sus diversas prácticas de enseñanza universitaria es la distancia entre la percepción de las exigencias del profesor antes y después de la evaluación. Las entrevistas y los balances del saber así lo indican.

Las dificultades halladas varían los estudiantes y su manera de reaccionar a las formas del saber enseñado. Luego, se confirma la hipótesis comprensiva y general de la investigación: la respuesta que dan los estudiantes a las exigencias de las formas de los saberes enseñados en la universidad dependen en gran parte de su relación con el saber, y de sus actitudes y tratativas o enfoques que esta relación implica. Se reitera la idea hipotética del carácter singular e individual de la relación con el saber en los estudiantes universitarios.

El estudio también arrojó otros resultados. Un conjunto de herramientas ${ }^{3}$ y elementos de naturaleza conceptual y práctica para llevar a cabo procesos de reflexión con el objetivo de examinar las prácticas de enseñanza y relación con el saber en los estudiantes de la educación superior. ¿A quiénes se dirigirían estas publicaciones? A los profesores universitarios que se preguntan o preocupan por sus prácticas, a los encargados o directivos de los cursos de formación de aptitudes pedagógicas universitarias, y a los consejeros psicopedagógicos de los estudiantes que existen en diversas universidades colombianas.

Se ha mostrado que el concepto de relación con el saber en la perspectiva adoptada en este estudio efectivamente está construido con cierto grado de coherencia tal que puede

3- Las herramientas son: (1) Los cursos en la enseñanza universitaria: conceptos para determinar su especificidad en la relación con el saber; (2) Los campos de limitaciones del saber enseñado en la educación universitaria y su relación con el saber; (3) Las dimensiones de las dificultades de aprendizaje de los estudiantes y su relación con el saber. 
tener un estatuto de concepto en el campo y ámbito en que se ha aplicado. Sin embargo, la expresión relación con (rapport à) de uso corriente a veces no se distingue suficientemente de otras nociones cercanas.

Así, Isabell Delcambre \& Yves Reuter (2002) consideran que el concepto relación con es, a menudo, tratado sin una construcción teórica suficientemente elaborada, y la evidencia de esta situación es la multiplicidad de vocablos o términos empleados (posición, lugar, postura, punto de vista, identidad enunciativa, relación con, incluso estatuto o rol) de manera vacilante. Situación que llevaría al concepto de relación con a ser un objeto donde cabe todo: la puesta en escena del sujeto, sus relaciones (con el saber, con el lenguaje, con la escritura, con las tareas) la implementación de estas relaciones, las diversas dimensiones que las estructuran (cognitiva, efectiva), etc. En ausencia de límites o de una construcción precisa de los componentes y su articulación, se corre el riesgo de una dilución conceptual.

A esta dilución conceptual se agrega el riesgo en el campo de las didácticas, que sería el de un uso sin precauciones de la noción para determinar las aptitudes de los alumnos en la educación media o de los estudiantes en la educación universitaria y encerrarlos de esta manera en una categorización, que para ser posible, necesita de investigaciones prudentes y complejas.

En efecto, al menos dos riesgos son posibles por una colusión entre el concepto de relación con elaborado teóricamente y un uso corriente de esta expresión: (a) por una reificación o cosificación de la noción, se puede rápidamente suponer que existe una relación con detectable a priori, sin tener en cuenta las situaciones concretas de la enseñanza y aprendizaje, en nuestro caso universitarias, lo que haría de la noción equivalente del hándicap socio-cultural, noción contra la cual fue precisamente construido el concepto de relación con: el interés del concepto reside precisamente en el hecho de no relacionar con el estudiante solo sus propias dificultades, sino tomar en consideración el contexto (escolar, universitario, entre otros) en el cual él evoluciona; (b) al querer determinar una relación con únicamente a partir de las producciones escolares de los alumnos y universitarias de los estudiantes, se corre el riesgo de confundirlas con una simple adecuación con las normas de la institución escolar o universitaria, planteadas $a$ priori como indiscutibles, con el añadido de un juicio evaluativo que no es compatible con el enfoque descriptivo y comprensivo, en nuestro caso, de la relación con el saber.

Finalmente, esta relación no implica solamente al estudiante universitario y sus aptitudes y comportamientos propios, sino también al profesor $\mathrm{y}$ a los otros actores del sistema universitario. Sin olvidar la posibilidad un principio que la relación con puede convertirse también en un contenido de enseñanza en la universidad. 


\section{Referencias}

ALZATE, María Victoria; DESLAURIERS, Jean-Pierre; GÓMEZ MENDOZA, Miguel Ángel. Cómo hacer tesis de maestría y doctorado: investigación, escritura y publicación. Bogotá: Ecoe, 2010.

ALZATE PIEDRAHITA, María Victoria; GÓMEZ MENDOZA, Miguel Ángel. Enseñanza y didáctica universitaria: discurso y formación documental del estudiante. Bogotá: Ecoe, 2009.

. La alegre entrada y el irresistible ascenso de las competencias en la universidad. Revista Educación y Educadores, Bogotá, Universidad de La Sabana, v. 13, p. 453- 474, 2010a.

. El "oficio" de estudiante universitario: afiliación, aprendizaje y masificación de la universidad. Pedagogía y Saberes, Bogotá, Universidad Pedagógica Nacional de Colombia, v. 33, p. 99-112, 2010b.

. Las tutorías en el contexto de las modalidades de acompañamiento pedagógico a los estudiantes en la enseñanza universitaria. In: HOYOS, Ana Julia (Ed.). Ponencias del encuentro internacional "Tutorías para la formación integral en la educación superior”. Medellín: Ministerio de Educación Nacional- Editorial Universidad de Medellín, 2010c. p. 81-105.

ALZATE PIEDRAHITA, María Victoria; ARBELAEZ, Martha Cecilia; GÓMEZ MENDOZA, Miguel Ángel. Enseñar en la universidad: saberes, prácticas y textualidad. Bogotá: Ecoe-Universidad Tecnológica de Pereira, 2011.

BAUTIER, Ėlisabeth; ROCHEX, Jean-Yves. L'expérience scolaire des nouveaux lycéens. Paris: Armand Collin, 1998.

BEILLEROT, Jacky. La formación de formadores entre la teoría y la práctica. Buenos Aires: Novedades: Universidad de Buenos Aires, 1998.

BEILLEROT, Jacky; BLANCHART-LAVILLE, Claude; MOSCONI, Nicole. Formes et formations du rapport au savoir. Paris: L'Harmattan, 1996.

BEILLEROT, Jacky; BOUILLET, Alain; BLANCHARD-LAVILLE, Claudine; MOSCONI, Nicole. Savoir et rapport au savoir: élaborations théoriques et cliniques. Paris: Éditons Universitaires, 1989.

BLANCHARD-LAVILLE, Claudine. Saber y relación pedagógica. Buenos Aires: Novedades: Universidad de Buenos Aires, 1996.

BROUSSEAU, Guy. L'échec et le contrat. Recherches, Nantes, n. 41, p. 177-192, 1980.

CHARLOT, Bernard. Du rapport au savoir: éléments pour une théorie. Paris: Anthropos, 1997.

CHARLOT, Bernard; BAUTIER, Élisabeth; ROCHEX, Jean-Yves. École et savoir dans les balieus...et ailleurs. Paris: Armand Collin, 1992.

COLOMBIA. Ministerio de Educación Nacional. ABC de la reforma a la educación superior en Colombia, 2012. Disponible en: <http://www.mineducacion.gov.co/1621/article-283356.html>. Acceso en: 7 de mayo 2012.

Ministerio de Educación Nacional. Deserción estudiantil en la educación superior colombiana: metodología de seguimiento, diagnóstico y elementos para su prevención, 2009. Disponible en: <http://www.mineducacion.gov.co/ sistemasdeinformacion/1735/articles-254702_libro_desercion.pdf>. Acceso en: 7 de mayo 2012.

COULON, Alain. Le métier d'étudiant: l'entrée dans la vie universitaire. Paris: PUF, 1997.

DE KETELE, Jean-Marie; ROEGIERS, Xavier. Méthodologie du recueil d'informations: fondements des méthodes d'observation, de questionnaire, d'interview et d'etude de documents. Bruxelles: De Boeck, 2009.

DELCAMBRE, Isabelle; REUTER, Yves. Images du scripteur et rapports à l'écriture. Pratiques, Metz, Cresef, n. 113-114, p. 7-28, 2002. 
DELEUZE, Gilles. Lógica del sentido. Barcelona: El Bote de Vela, 1990.

HOUGARDY, Philippe; PAMBU KITA, Phambu. Dispositifs de remédiation à l'université: pour quels étudiants et avec quel bénéfice. In: LOURYAN, Stepane; THYS-CLEMENT, Françoise. (Dir). Enseignement secondaire et enseignement universitaire. Buruxelles: Editions de l'université de Bruxelles, 1999. p. 77-92.

MILES, B. Mattew; HUBERMAN, Michele. Analyse des données qualitatives. Bruxelles: De Boeck, 2003.

MORANDI, Franc; LA BORDERIE, René. Contrat pédagogique. In: Dictionnaire de pédagogie. 120 notions clé 320 entrées. Paris: Nathan, 2006. p. 112-113.

POLLET, Marie Christine. Pour une didactique des discours universitaires. Bruxelles: De Boeck, 2001.

POZO, Juan; ECHEVERRÍA, María Del Puy Pérez. Psicología del aprendizaje universitario: la formación en competencias. Madrid: Editorial Morata, 2009.

REY, Bernard. Diffusion des savoirs et textualité. Recherche et formation, Lyon, n. 40, p. 43-57, 2002.

Peut-on enseigner la problématisation? Recherche et formation, Lyon, n. 48, p. 91-105, 2005.

ROMAINVILLE, Marc. Savoir parler de ses méthodes. Bruxelles: De Boeck, 1993.

Savoir parler de ses méthodes: métacognition et performance à l'université. Bruxelles: De Boeck, 1999.

L'échec dans l'université de masse. Paris: L'Harmattan, 2000.

Recebido en: 04.04.2013

Aprobado en: 27.06.2013

Miguel Ángel Gómez Mendoza es profesor en la Universidad Tecnológica de Pereira, Colombia.

María Victoria Alzate Piedrahita es profesora en la Universidad Tecnológica de Pereira, Colombia. 